\title{
1.1
}

\section{COMMUNICATING BIG THEMES IN APPLIED MATHEMATICS}

\author{
Julian Hunt FRS \\ Lighthill Institute of Mathematical Sciences and \\ University College London, UK \\ (and JM Burgers Centre, TU Delft, The Netherlands)
}

\begin{abstract}
This paper reviews, primarily for mathematical educators, how decisions taken in government and the private sector make use of the concepts and techniques of mathematical sciences, particularly those related to sudden and extreme events in natural, social and technological systems. The insights of applied mathematics, statistics, and computational modelling are shown to be relevant to understanding and managing the outstanding problems concerned with these systems. The main themes highlighted here include predictability, accuracy, extreme values, singular events, and patterns in system behaviour, especially in relation to new approaches in deterministic and statistical modelling and to the increasing use, through computer networks, of the vast volumes of data that are now available to scientists and decision makers. These developments also exploit improved optimisation methods which make better use of approximate data and, most recently, the 'multi-centre' combinations of model results obtained at different institutions, which benefit from the slightly different assumptions and numerical methods at each centre. Suggestions are made about how these concepts and techniques could be used more effectively in government and industry to guide both tactical and strategic planning. The worlds of media and politics also need to understand better how mathematically-based predictions are made and how to question them. Universities could provide general 'appreciation' courses to survey the general ideas of mathematical science and their wide applicability in society. Graduating mathematical scientists might be encouraged to take on more responsible roles which should be of benefit both to their organisations as well as to their own careers.
\end{abstract}

\section{MATHEMATICS AND SOCIETY'S MAJOR CHALLENGES}

Applied mathematics and mathematical modelling are increasingly vital for understanding and dealing with society's major challenges. This is slowly being recognised, even by policy makers and industrialists, as they base more of their decisions on the concepts and calculations of mathematical sciences. Correspondingly, they rely less exclusively on expedience, experience, and intuition. This transition has been helped by the increasing use of graphical presentations, for example, with PowerPoint displays; though rhetoric still has its place. (Of course, through public opinion polling, even the most ancient of political techniques can be improved through mathematics!) The general public, as well as journalists, are 
increasingly using mathematical language and images, the current favourites being chaos and tipping points. These may change as pattern recognition, now part of the school curriculum in year 6 , and self organisation become more relevant, for reasons discussed in this paper. The increased public recognition of the conceptual importance of mathematics has not come about by chance. The whole community of mathematical scientists throughout the world is making progress in communicating advances in research and their practical implications, but also how these developments affect the way we look and think about the world around us. In the UK, many organisations are contributing, particularly the mathematical societies (the Institute of Mathematics and its applications, the London Mathematical Society, the Royal Statistical Society) and institutes of mathematical sciences in universities, such as the Lighthill Institute of Mathematical Sciences, Smith Institute etc. However governments, while recognising the value of mathematics for industrial applications and statistical analysis (for example, Hunt \& Neunzert, 1993), have still not understood their wider implications for societal problems.

Two major themes of mathematical science are discussed in this paper, where progress in research has made a particular contribution. The first is the analysis and description of extreme and singular behaviour, particularly how, where and when sudden changes and isolated events can occur in human and natural systems. The second is the behaviour and the limits to the capabilities of computation and data systems as they affect the accuracy and reliability of prediction of multi-component, complex systems. Related advances in mathematics and computer science have also led to the improvements of the control of such systems, especially in complex and changing circumstances.

The issues of greatest public concern can be identified where these themes are particularly relevant for policy and public understanding:

- Natural and technological disasters and consequences for communities, including tsunamis, earthquakes and hurricanes (2005 was a particularly bad year; Hunt, 2005, 1995; Huppert \& Sparks, 2006).

- Large changes in natural systems where key components change more or less suddenly; from one state to another or through a major perturbation which eventually decays, for example, climate change events (with permanent or longterm global consequences) or global epidemics (which usually decay) (Schellnhuber et al., 2005).

- Isolated perturbations to local systems (strikes, acts of violence, etc.), for example, analysed qualitatively using catastrophe theory (Zeeman, 1987).

- Large, possibly sudden, changes to weakly-constrained or malfunctioning interacting systems (for example, Enron business collapse), or the initiation or the cessation of conflict, as occurred with the end of the Cold War, which LF Richardson predicted by his simple mathematical model in 1953. (see Hunt, 1993, 2001). However, Richardson warned that excessive reliance on mathematical models can be very misleading. The best known failure was that of the US Defence Department's models of 'pacification' in the Vietnam War.

- Improvement of performance of economic and social organisations in response to political and financial inputs and operational constraints. Mathematical analysis can test the statistical measures used by policy makers and the effects

Mathematical Modelling (ICTMA12) Education, Engineering and Economics ISBN 978-1-904275-20-6 Chichester: Horwood (2007) 510pp.

Editors: Christopher Haines, Peter Galbraith, Werner Blum and Sanowar Khan. 
of specific targets on complex organisations, especially where the organisations, including governments, are aiming to integrate or 'join up' their activities. (Hunt, 1997; Gallivan, 2006).Concepts and models of organisations models have to allow for the unpredictability caused by random and chaotic effects, replacing earlier technocratic concepts of society running like a well oiled engine-the image of the German engineer Reuleaux (1885)

- $\quad$ Prediction and design of network systems (capacity, performance, robustness etc.; transport; internet...), where new solutions to new problems and new demands have to be considered (for example, on line response, security, data compression/ communication) (Kelly, 1979).

- Understanding and redesign of physical systems (houses, cities, products) to meet new constraints; how to invent and define total changes in their structure and patterns of performance both conceptually and by massive computational modelling (Lautso et al., 2004). In electronics, new physical and mathematical concepts are developing both for the 'end' of silicon technology and of deterministic, non-quantum computing, as the size decreases and the speed increases of current solid state devices (House of Lords, 2002).

\section{EXPLAINING THE INSIGHTS AND THE LIMITATIONS OF APPLIED MATHEMATICS AND MODELLING}

Mathematical analysis and modelling is evermore widely used by policy makers and industrialists for guiding and even making their decisions. However, by asking some basic questions they can understand better what reliance to place on the results, and how to interpret them. They also want to know the likely future trends of these techniques.

For mathematical scientists, identifying and explaining the general concepts that emerge from their particular studies is an essential part of making progress in research. As Henri Poincaré (1914), probably the best philosopher of applied mathematics, first explained, generalising the results of a particular study enables scientists to move from one hypothesis to the next and to relate the research to wider scientific developments. In expounding his philosophy as a biologist, Haldane (1935) argued, following Kant, that the connectedness of scientific concepts means that science is not simply a subjective creation, like art, but has an objective validity. This philosophical insight is related to the sociological fact that people's familiarity with scientific data and concepts (for example, weather forecasts) enables them to make their assessments through their engagement with scientific data. One might conclude that only through such familiarity can people understand scientific concepts and be more inclined to accept the validity of controversial science.

In the UK, government funding of research implicitly recognises that this generalisation and interconnection of scientific understanding, and its popularisation, is necessary for building up an integrated structure of scientific knowledge. It is also necessary for ensuring that over the long term there is continuing support for science by society as a whole. 


\subsection{How are Different Types of Models Constructed, Corrected and Rejected?}

Reductionist models used for predicting well-defined physical systems are built up from the basic laws of physics, chemistry. However, for predicting complex and larger scale systems, such as rockets or the weather, the models generally involve approximations and some empirical assumptions. The reductionist approach is also used for predicting the behaviour of certain types of economic, social and biological systems. However, here the models are based on ad-hoc hypotheses deduced from empirical observations, for example, about animals' and people's greed, aggressiveness or submissiveness as in predator-prey models (Volterra, 1931) or driver reactions in traffic models.

The alternative approach for modelling the behaviour of complex systems is statistical, which is based on the assumption that it can be calculated from data describing its previous behaviour. Generally past statistics do not allow for changing conditions. But in some cases when the future is not the same as the past (for example, river floods in a changing climate (Hunt, 2002, 2005) corrections can be introduced, sometimes involving a combination of statistical and reductionist models.

Both approaches essentially began in the $17^{\text {th }}$ Century, when Newton introduced dynamical deterministic models and Pascal, Leibniz and Bernoulli invented probabilistic prediction (Favre, 1995; Hacking, 1974). These models, especially the former, have steadily improved, and grown more complex mathematically as they represent and predict more complex processes, more detail, more components and more dimensions in space, time and phase space. As more computer power becomes available, the speed, complexity and data input are continually increasing. However, many processes have a chaotic aspect of their behaviour associated with extreme sensitivity to small changes, which may be imposed by external conditions or may be intrinsic to the model or the actual process. These aspects may or may not be predictable in advance! However with recent developments in mathematical science and increased computer power, the calculation of all the theoretical possibilities of ideal systems becomes possible. This provides increasing insight into chaotic behaviour of processes, their representation by models and their dependence on data.

Although there is still no rigorous framework for combining different methods, such as statistical and deterministic analyses for modelling complex systems, empirical approaches are being developed for many types of practical problem. In particular statistical science, which is largely based on methods for analysing empirical data, is now being applied to analysing the computed outputs of deterministic models whose randomness results from the measured and assumed distributions of the inputs to these models.

Despite theoretical and conceptual problems arising from such hybrid methods, Maxwell and Boltzmann in the $19^{\text {th }}$ century pioneered this approach with their study of molecular dynamics and random systems. In the $20^{\text {th }}$ century, Wiener, Kolmogorov and others brought statistical concepts into dynamical models, for example, for turbulent flows (for example, Landau \& Lifshitz, 1960).

Reductionist models with empirical elements are widely used in oceanography and meteorological models for forecasting particular events. But they cannot be run for long enough to provide reliable statistics of future likely events at any particular

Mathematical Modelling (ICTMA12) Education, Engineering and Economics ISBN 978-1-904275-20-6 Chichester: Horwood (2007) 510pp.

Editors: Christopher Haines, Peter Galbraith, Werner Blum and Sanowar Khan. 
location. Nevertheless, in combination with empirically based statistical models, (for example, of future storm events in a changing climate), reductionist methods provide computer estimates for the probabilities of future sea level variations around the UK coast. This combined approach is regarded as more comprehensive and more reliable than simply making predictions based on past tidal records (Butler et al., 2005).

Both reductionist and statistical models are now being combined in financial and risk models. The analytical results and numerical simulations provide statistics that contribute to decisions in insurance, investment. (Turfus, 2006).

For calculating specific results in the 'real world' both of these types of models require data to initiate the calculation (for example, today's stock-price or weather) and to define the range of conditions where they are relevant. The essential point is that in the 'real world' since there is insufficient data, assumptions have to be made about the missing data.

Since the 1980 's, new techniques of 'data assimilation' and data optimisation have been developed which have utilised data much more effectively to improve the accuracy of deterministically based models, and reduce errors caused by missing data. The first step was to make the optimum adjustment of the predictions as new data is received. Eventually the 'best line' is drawn through the data points consistent with the 'dynamics' of the system. In more advanced procedures, the previous computations are repeated as new data is provided. These techniques enable the vast quantity of data, now available for monitoring and scientific modelling, to be used much more effectively, notable for weather forecasts using satellite data (Hunt, 1999; Hunt \& Coates, 2002).

These data assimilation concepts are beginning to be applied to the prediction and control of flows in aeronautical engineering (Bewley, 2003). Many other applications are also possible.

Understanding the errors of models and having estimates for the reliance that can be placed on their prediction, are both essential for their use in decision making.

Model predictions have markedly different patterns of growth with time. They fall into four main categories.

a) A nearly perfect type of forecast, such as these associated with regular planetary motion where the errors do not increase with time.

LF Richardson (1922) thought wrongly that weather forecasts might reach this ideal given sufficient precision in numerical methods and in the measurement data that initiate the calculations. Nevertheless some geophysical systems are highly predictable as the recent calculation of the global movement of the tsunami waves in 2005 , were remarkably accurate as they travelled on curving paths half the globe.

b) For a forecast of a typical non-linear system of limited complexity with time scale $\mathrm{T}_{\mathrm{s}}$ (i.e., with a few degrees of freedom), errors tend to grow exponentially (i.e., proportional to $\exp \left(\mathrm{t} / \mathrm{T}_{\mathrm{s}}\right)$ ). Therefore, the calculations are very sensitive to initial conditions, as first explained systematically by Lorenz (1963) though hinted at earlier by Poincare (1914). The chaotic nature of the time variation of simpler mechanical systems is described by

Mathematical Modelling (ICTMA12) Education, Engineering and Economics ISBN 978-1-904275-20-6 Chichester: Horwood (2007) 510pp.

Editors: Christopher Haines, Peter Galbraith, Werner Blum and Sanowar Khan. 
Moon (2004).

c) The predictability of complex non-linear systems (with very large numbers of spatial and temporal degrees of freedom) is different when they organise themselves into coherent and persistent patterns of behaviour. Examples of discrete and continuous systems range from galaxies and atmospheric eddies in the physical universe and from societies to biological organisms in the living world. Although these patterns are randomly distributed in time and space, they have a finite life -time and spatial scales (defined mathematically by correlation functions). Time scales are shorter if each element of the system is changing rapidly or is dissipating a lot energy (whether physical or human). The persistence of such patterns means that errors in prediction grow more slowly with time than in type (b) (typically in proportion to some power of time $t^{\alpha}$ ). For the errors of location of large scale weather systems, as well as magnitude of variables such as pressure and temperature, the value of the exponent $\alpha \simeq 1$ (Met Office, 2003; Hunt, 1999).

d) The most feared and least well predicted features of the overall or average behaviour of systems occur when they have a large and sudden change, typically from one state to another (see $\S 2.4$ ). These 'catastrophes' or 'bifurcations' can occur over periods much shorter than the time-scale of the usual fluctuations of the system. The errors in predicting such changes also tend to have a 'step- function' variation with time. Examples abound in both in physical systems (for example, earthquakes, volcanoes) and socio-economic systems where the events were not predicted at all, as with the dramatic ending of the Cold War in 1989.Their timing and magnitude tend to be equally erroneous. In many situations there are indications that changes are about to occur, but when warnings have been given, they have often been ignored, perhaps because they have been uncertain or so unexpected. Furthermore, they are as likely to be ignored whether they are optimistic as pessimistic, as was the view of Richardson's prediction in 1953 that could be a sudden end of the arms race and the Cold War. (Hunt, 2001).

Even in well defined laboratory systems these kinds of bifurcations can be unpredictable. Typically, they are found when the initial and boundary conditions are symmetric and do not define a preferred orientation, such as occur in buckling a solid structure or in thermal convection produced by heating initially stationary liquid in a long horizontal container. In this case, turbulent motions tend to induce a persistent mean flow whose direction (Krishnamurthi \& Howard, 1981; Owinoh et al., 2005) is unpredictable. Bifurcations are inevitably sensitive to small external perturbations. Since similar kinds of mechanism can affect large regions of the ocean, the global climate could also change rather suddenly (as it has done in the geological past).

There is no doubt that in recent research on non-linear systems there has been considerable progress in their prediction using deterministic (and in some cases statistical) models, especially where the systems are the self-organising type (c). As

Mathematical Modelling (ICTMA12) Education, Engineering and Economics ISBN 978-1-904275-20-6 Chichester: Horwood (2007) 510pp.

Editors: Christopher Haines, Peter Galbraith, Werner Blum and Sanowar Khan. 
a result of more detailed and accurate computations, these models now include more physical processes and more variables (for example, some aerosol effects in global climate models), and also more comprehensive measurements to initiate and correct the calculations, for example, through satellite monitoring of weather systems, or survey data of economic and social system. Indeed, as with the example of the tsunami wave, some calculations of type (c) are now so successful that they now fall into type (a).Another example of progress is that weather forecasts for 3 days are now as accurate as 1 day forecast 20 years ago in the North Hemisphere and about 10 years ago in the South Hemisphere (when reliable satellite data were not available) (for example, Hunt \& Coates, 2002).

Empirically based models are used for predicting the economic growth rate for 12 months in advance. Although the variations between models are as much as $100 \%$, in recent years, they have become more reliable (at least for developed countries). Perhaps this only because there have been no major economic perturbations (such as occurred in Asia in 1998 and in UK in 1992)

The history and philosophy of science has only recently begun to describe how scientific concepts and models really evolved. The basic idea taught in textbooks is that hypotheses are tested and then accepted or rejected depending on how well they compare with experimental data; provided, as Karl Popper pointed out, the hypotheses are in a form that can be disproved. This traditional interpretation of science dominates most journalists' discussions of scientific developments, leading them to the naïve conclusion that any discrepancy with data should lead to rejection of scientific theory. In fact, as Lakatos first explained, science proceeds more smoothly, with models being progressively corrected to allow for discrepancies that are bound to occur. This is an essential part of scientific progress (Chalmers, 1982). An important recent example was the correction of the first global climate models around 1990 which predicted an excessively large rise in the global temperature as a result of the increase in green house gases emitted by human activities. The model was corrected to allow for the industrial aerosols which reduced the incoming solar radiation at ground level (Hunt, 1999; Houghton, 1994).

Occasionally new data becomes available that can only be explained by a new conceptual approach and a rejection of current models, as occurred with the revolutionary changes in Newtonian and Einsteinian physics. Kuhn defined these events as 'paradigm shifts' in science. Lorenz's (1963) demonstration of the unpredictability of quasi-deterministic systems was indeed a paradigm shift in applied mathematics.

\subsection{Extremes}

In the practical use of models questions can arise as to whether the models are also applicable when the system experiences extreme conditions. If the answer is no, is it possible to extend models designed for 'normal' conditions to include these events? Or will it be more accurate and more computationally efficient to rely on special models for extreme conditions?

The usual calculations for the safety of engineering structures are based on the analysis of small static deflections. These are clearly inappropriate for assessing the effects of large unsteady loading and intense temperature caused by an aircraft

Mathematical Modelling (ICTMA12) Education, Engineering and Economics ISBN 978-1-904275-20-6 Chichester: Horwood (2007) 510pp.

Editors: Christopher Haines, Peter Galbraith, Werner Blum and Sanowar Khan. 
crashing into it and exploding. Arguably, accurate calculations for such rare disasters are unnecessary. But they do matter if the destruction has damaging consequences on the surrounding environment - an essential consideration for designing nuclear power stations, when extreme conditions have to be modelled in some detail. Indeed, in this case, the extreme conditions are so important that they determine the overall design. The same dichotomy is apparent in modelling many types of extreme situation; whether they are natural disasters such as floods, and large earthquakes, or economic/ social calamities such as wars, slumps, riots etc.

It is a remarkable fact that for extreme events, which occur rarely, randomly and in an unconnected way in completely different system, their frequency of occurrence and magnitude tend to be described by general statistical laws. LF Richardson showed how the Poisson distribution (which describes frequency of horse kicks killing Prussian soldiers), also describes the occurrence of wars (see Ashford, 1985). For these and most other extreme events, determining the appropriate form and parameters of the statistical distribution depends to a great extent on the analysis of limited data sets of extreme events. Recent research has shown how sensitive the resulting distribution is to the analysis; for example, estimates for the return period of floods may change from 50 years to 10 years when data is taken only from the most extreme previous events (Cox, Isham \& Northrup, 2002; Embrechts et al., 1997).

While statistical models provide guidance about the frequency and therefore the likely occurrence of an extreme event, what actually happens during the events can only be predicted by deterministic models. In wind storms, floods, and tsunami waves, the intense air and water motions vary over much shorter distances than in normal conditions. In hurricanes the winds vary over a few kilometres, whereas in usual low pressure systems they vary over hundreds of kilometres. Special models now forecast hurricane trajectories in real time (usually for 5 days ahead but up to 10 days in some situations). However, it is not necessary to solve the basic equations to make a forecast, because the events are sufficiently similar (as satellite images demonstrate so clearly) that the same special (vortex) model can be used for the inner structure of hurricanes, while the wind far from the centre is the forecasting model used for normal conditions (for example, Heming et al., 1995). Progressively, these special methods may be replaced by models used for normal conditions as computer power increases, and general calculation methods adapt to events on small length and time scales (Nikiforakis \& Hubbard, 2003).

The frequencies of extreme events in complex systems tend to have characteristic statistical distributions such as the occurrences of hurricanes or tropical cyclones for each area of the world. These frequencies differ from the distribution of high winds in general. However, extreme events of one kind of phenomenon may be correlated with other related phenomena. In the case of hurricanes, physically based models show that their movement in any given season is affected by the prevailing winds. Using this information predictions for annual hurricanes reaching land have recently become significantly more accurate (with errors of less than $20 \%$ in any given zone) (Saunders, 2005).

However, as with all statistical models they are based on measurements of past events, so they have to be modified if the conditions affecting the random events are changing with time. A combination of deterministic models for global climate

Mathematical Modelling (ICTMA12) Education, Engineering and Economics ISBN 978-1-904275-20-6 Chichester: Horwood (2007) 510pp.

Editors: Christopher Haines, Peter Galbraith, Werner Blum and Sanowar Khan. 
prediction (described above) and these statistical models are now being used to allow for the effects of climate change on probability estimates of extreme weather events. But the results depend crucially on the modelling of physical variables in the statistical models. The result of these studies show how in a warming global climate some of these factors increase the strength and frequency of storms, while others reduce them. The consequences for the insurance industry are obviously considerable!

\subsection{Patterns, Shapes and Approximate Representations}

Nowhere has the impact of mathematical science on modern society been so marked as its provision of techniques for defining characteristic features of signals of every kind. Fourier's series representations of signals in terms of oscillations in the early 19th century (see Farge et al., 1993) and Luke Howard's (1803) verbal and physical classification of cloud shapes were break-through concepts we still rely on. But equally revolutionary changes in the $20^{\text {th }}$ century occurred with the discovery of the fractal dimensions of wiggly shapes (for example, Mandelbrot, 1982) and the quantification of the information contained in a signal and its efficient transmissions by Shannon \& Weaver (1949). Scientists, engineers, economists and social scientists were able to educe or detect critical features hidden within complex signals and at the same time transmit the signals more efficiently by focussing on these features. These developments enable models of complex systems to be assessed more efficiently in terms of other models or their accuracy against measurements; rather than analysing many cases and huge volumes of data, only the key aspects of the measured or calculated signals have to be compared.

Even our aesthetic appreciation of shapes and surfaces has been changed by mathematical signal analysis, especially through the computer graphics of fractal boundaries, and the depiction of complex spiral shapes. Patterns of fractal laser light beams reflected off clouds at parties round the world show how the aesthetic aspects of mathematics can be a universal language enjoyed by everyone!

In every academic discipline major discoveries have resulted from developments in the mathematical analysis of data. The results may well be quite controversial depending on the choice of the techniques applied and in some cases how they are combined.

In geo-physics, the modern understanding of the structure of the earth's core resulted from analyses of earthquake waves, In astrophysics by analysing the pattern of microwave radiation beyond the outer-most stars it has been possible to deduce that the earliest stage of the universe was not formless - a hypothesis with the profoundest cosmological and even religious implications!

The analysis of complex data gave rise to an equally revolutionary change in ideas in social science in the 1920's (Spearman, 1904; Garnett, 1921). From sets of cross-correlations of the results from various psychological tests, matrices were constructed. It was postulated that their eigenvectors, or principal components, provided an overall measure of 'intelligence', in a similar way that the eigen vectors of oscillating or deforming mechanical systems define intrinsic properties that are independent of any particular reference frame for the measurements. The controversy surrounding of the application of these standard mathematical methods 
continues to this day. Eigenvectors of climate patterns are now relied upon to predict seasonal weather for several months ahead (Colman \& Davey, 1999).

Since different mathematical education techniques tend to focus on different features of a signal, there have been controversies about what the most critical features are for any given signal under varying circumstances. Some techniques analyse the entire data set, while others select only certain data (for example, above a certain threshold).This is typically what animals -including humans- do in processing sight and sound. Fourier (or spectral) methods compare the signals to sinusoidal oscillations, while other (for example, fractal or capacity dimension methods) describe these in terms of the gaps (in time or space) where the magnitude of the signals are below a certain threshold. Research (for example, Bonnet \& Glauser, 1993) has shown both theoretically and practically how the relative merits of different identification techniques are better understood, so that when applied to fluid mechanics, they now tend to agree about the general form of the characteristic three-dimensional time-varying 'eddy' patterns in the turbulent flows - in a sense vindicating Howard's hypothesis of 1803.

Much of modern technology depends on rapid communication of huge quantities of data using miniaturised transmissions and receiving systems. In the mobile phone systems, images, speech and visual data are digitised and effectively Fourier analysed into waves or on-off signals. Such data used to be 'compressed' rather crudely with the aim of retaining only its essential features by simply excluding high or very low frequency components of the signals. But a more discriminatory approach is to follow the information system the eye and brain and focus on the 'edges' in a signal where sharp changes occur in the amplitude (Farge et al., 1993; Silverman \& Vassilicos, 1999).

Dealing with sharp gradients, such as shock waves and cracks in solids, is also a challenge. In the computational models, the methods developed earlier for concentrating numerical meshes around these critical regions are now being extended to more complex problems where the critical regions are randomly distributed and also move randomly in time (from chemically and biologically active media to weather and climate models (for example, Hubbard and Nikiforakis, 2003).

\subsection{Singular Events and Sudden Changes}

People have to decide what to do about sudden or even singular events in their lives, whether they are managers or employees in organisations, or driving a car through traffic jams, or more seriously when confronted by an accident or natural disaster. Well prepared individuals and organisations need to know in advance what kinds of events might occur in 'their' system, and when and how they happen, and what they should do.

A mathematically informed approach can certainly help, especially as governments and communities become more aware of all the kinds of risks they need to prepare for. They also have to consider the possibility of several hazardous events occurring close together in space and time - a precaution that is becoming increasingly necessary in modern communities whose compactness and interdependence makes them more susceptible to such events.

In some situations (discussed already in $\$ 2.3$ ), precise modelling of the events

Mathematical Modelling (ICTMA12) Education, Engineering and Economics

ISBN 978-1-904275-20-6 Chichester: Horwood (2007) 510pp.

Editors: Christopher Haines, Peter Galbraith, Werner Blum and Sanowar Khan. 
may be possible. Also they may occur with sufficient regularity that their future probability of occurrence can be estimated quite reliably. But for events in a changing environment (for example, climate change or larger life span), systematic studies of the underlying trends and mathematical modelling may be the best way of predicting the nature and frequency of extreme events.. In some cases these fundamental studies have indeed predicted the form of changing extreme events before they were observed (as a hurricane has now occurred in the South Atlantic, in 2005 , for the first time).

Mathematical analyses of events begin by defining a variable $\mathrm{V}$ as a function of one or more independent variables., There might be a 'step' change in traffic flow along a road (in fact over the few seconds corresponding to drivers' reaction times). In an idealised mathematical analysis this period is considered to be 'infinitesimally' small, so that the variation of $\mathrm{V}$ is approximated by $\mathrm{H}(\mathrm{t})$ the Heaviside step function.

This implies that the rate of change of $\mathrm{V}$, i.e., the acceleration or deceleration, becomes very large. Over the infinitesimally small period $\mathrm{dV} / \mathrm{dt}$ is now approximated by the 'spike' or Dirac's 'delta' function $\delta(\mathrm{t})$ - probably the most famous development of mathematics in the $20^{\text {th }}$ century (Lighthill, 1953).

New types of highly oscillatory singularity that have been conceived mathematically also correspond to observations. Random and multi-dimensional functions are other features of more complex singularities. Such idealised mathematical functions are particularly useful for describing measured variables near the points at which the functions are singular, (for example, $x_{0}=x_{\circ}$ ) in the above example. But very close to the singularity itself at $\mathrm{x}=\mathrm{x}_{\circ}$ (where $\mathrm{V}$ and/ or its derivative tend to infinity) the measured variables cannot exactly correspond to the idealised function. Many extreme events occur are singular in both space and time, i.e., the magnitude of the spatial and temporal derivatives, $\partial \mathrm{V} / \partial \mathrm{x}, \partial \mathrm{V} / \partial \mathrm{t}$ are very large at $x_{\circ}$ and $t_{\circ}$. Earthquakes form through the sudden rupture of solid materials along lines of weakness. In such cases, predicting the location $\left(\mathrm{x}_{\circ}\right)$ of where sudden large displacements $\mathrm{V}(\mathrm{x}, \mathrm{t})$ might occur is usually more certain than the moment $\left(t_{\circ}\right)$ when they might occur, as are the recent major earthquakes of Kobe, Sumatra and Kashmir so tragically demonstrated. In these events, the amplitude V (i.e., speed of the displacement) rapidly, but monotonically, increased to a maximum and then decreased.

But there are many types of singularity where the sudden amplification of V (or $\mathrm{dV} / \mathrm{dx}$ ) is associated with high frequency oscillations and waves. Deep vibrations within the earth, which were observed to amplify before the huge volcanic eruption of Mount Pinatubo in 1992, helped provide a vital warning which saved many lives (Hunt 1995). In London the notorious wobbles of the new footbridge across the Thames in 2000, which delayed its opening, was a vivid example of how oscillatory systems and people's behaviour can interact strongly as one effect feeds 'back' on the other - phenomena familiar to economists and politicians.

Many sudden events are also followed by waves and oscillations, as occurs in the Tsunami and hurricane induced waves in 2004/5 (Hunt 2005; Huppert \& Sparks, 2006). The moving blockages on motorways and in production systems 
(Armbruster, 2006) (both modelled by Lighthill \& Whitham's (1952) kinematic wave theory) are notable examples of how physical systems and people interact, sometimes with similar patterns (not unlike Richardson's concept of conflict).

Of course most sudden and singular events are multi-dimensional; the beautiful, but potentially dangerous events of huge breaking waves or atmospheric cloud billows, as they develop sharp crests that curve into spiral forms, have to be described in two space dimensions ( $\mathrm{x}, \mathrm{y})$ and time ( $\mathrm{t}$ ). Thom's catastrophe theory (for example, Saunders, 1986) is a mathematical framework for describing similar types of multi-dimensional singularities, but for most physical systems it has not provided any predictive insights. However, Zeeman (1987) suggests that certain singular events in prison break out and in hostage situations (described by a set of behavioural variables) might be predicted using these concepts. This approach has not been taken further.

In economics, oscillations and singularities are also analysed in great detail. In the 1990's some national economies experienced sudden and sharp reductions in output. For major economies, there is a continual debate as to whether the recovery of national economies from minor depressions are monotonic or oscillatory, or as the journalists say are 'double dip' events. The lessons from these extreme and sometimes sudden events, in many types of systems, is that through continued and close monitoring and studying of data, sudden events can lead be predicted. The growing fluctuations in share prices before the October 1987 'crash' and in the information 'chatter' on the internet preceding the terrorist event in New York in 2001 demonstrated such linkages. They were noted afterwards, but the data were not heeded beforehand.

When information about impending natural disasters is disseminated and used proactively it can save lives and it costs much less than dealing with the aftermath. Damage caused by these events is generally reduced when warnings have been issued in advance. This is why governments are finally accepting the need to improve the international warning systems and follow up arrangements (ISDR, 2005).

The most disruptive disturbances to complex systems lead to changes to different states of the system; subsequently they may or may not revert to the previous state. If they do, how long does this take? These are questions that can be examined using the ideas of mathematical dynamical system's theory, which for example can define particular situations when large changes are likely and what form they might have. Policy makers need to study these questions very thoroughly, since too often it is assumed that the state of a system before the disturbance is the only possible and desirable state, despite the numerous counter examples in engineering, biological, geophysical and political economy.

The mathematical description of a system's behaviour can be expressed using a phase-plane diagram in which one dependent variable (say $V_{X}$ ) is plotted as a curve against another (say $\mathrm{V}_{\mathrm{y}}$ ) ( such as a variable $\mathrm{V}$ and its derivative $\mathrm{dV} / \mathrm{dt}$ ) over the range of values of the independent variables $\mathrm{x}, \mathrm{y}$ or $\mathrm{t}$ or the parameter $\mathrm{p}$ (for example, Bondi, 1991; Thompson \& Stewart, 2002). The mean or characteristic features of a complex system could be described by the statistics of these curves (for example, average values or values in certain particular or 'conditional' situations).

Mathematical Modelling (ICTMA12) Education, Engineering and Economics ISBN 978-1-904275-20-6 Chichester: Horwood (2007) 510pp.

Editors: Christopher Haines, Peter Galbraith, Werner Blum and Sanowar Khan. 
The simplest examples are the circular curves of $\mathrm{V}_{\mathrm{X}}$ against $\mathrm{V}_{\mathrm{y}}$ describing flow pattern of eddy motion in the laboratory, the cooking pan or the atmosphere. Similar curves of $\mathrm{V}$ against $\mathrm{dV} / \mathrm{dt}$ describe time varying oscillations. In some cases these

flows can flip from one direction to another, either unpredictably and spontaneously, or as the parameter $\mathrm{p}$ changes. By using the powerful concept of symmetry, these types of bifurcations or global instability can be characterised as a jump from one region to another in the phase plane diagram (for example, Holmes et al., 1996). Many models of biological systems such as those for population of predators and their prey show how their behaviour can fluctuate around an equilibrium state (as in the case of arctic foxes and hares). But when external conditions of a system are disturbed too severely, the system changes utterly to a new state (possibly with either only predator or only prey or neither).

In politics and economics, the language of non-linear dynamical system has gradually tended to supercede the earlier linear control theory or cybernetics (or steering) models. President Nixon famously described his economic policy method in 1968 as a 'light touch on the tiller'. In the 1960's and 1970's when it was argued by the politicians in the UK (on both the left and the right) that the economic 'system' should and could move to a new 'state'. In reality the quite sudden fluctuations and 'stop-go' policies of national economies of various countries indicated the limitations of central control. By the 1990's the concept of a lightly controlled, but largely self-equilibrating market with its characteristic oscillations (i.e., the business cycle) became the accepted model. Prof Desai of London School of Economics has remarked on the fundamental difference between forecasts of weather and of economic systems, because governments that make the forecasts about the economy, can subsequently influence it. So an accurate forecast probably needs to allow for the deliberate actions of people and organisations or the system that might be affected by the forecast. On the other hand, governments cannot control the weather (and, so far as is known, gods of weather do not listen to the forecasts!). In fact, the previous lack of success of economic planning and the inaccuracy of the associated forecasting led the government in a number of countries (including the UK) to reduce their control of key elements of the system (i.e., bank rate).The forecasts seem to have become more accurate as a result of this decoupling of forecasting and control.

\subsection{Optimisation}

For designing and controlling the behaviour of systems to satisfy some optimum criteria, for example, of maximum performance or minimum cost subject to certain constraints, it may not be necessary to calculate every one of its possible states as a means of finding the relevant ones to meet the objectives. Other more direct methods may be available for directly calculating the 'optimum' behaviour. These methods are especially relevant when input data are insufficient to define the behaviour of the system; some data may also be mutually inconsistent.

For perfect systems, with perfectly prescribed data, optimisation calculations can lead to results that are equivalent to those obtained by modelling deterministically the detailed behaviour of the system. However, for imperfectly defined systems and

Mathematical Modelling (ICTMA12) Education, Engineering and Economics ISBN 978-1-904275-20-6 Chichester: Horwood (2007) 510pp.

Editors: Christopher Haines, Peter Galbraith, Werner Blum and Sanowar Khan. 
imperfect data, optimisation methods can have marked advantages for predicting system behaviour, essentially because such calculations integrate the overall behaviour and are much less perturbed by small errors. But where the system is in a state that is very sensitive to initial conditions, imperfect data can certainly lead to large errors for example, in predicting localised extreme events.

Optimisation methods were first used for analysing continuous systems determined by physical laws (for example, fluid, solid or electromagnetic). In the $18^{\text {th }}$ Century, Euler and Lagrange discovered that the Newtonian differential equations models for mechanical or planetary systems governed by law of dynamics (for velocity and force variables) were mathematically equivalent to finding the minimum of an integral of the kinetic and potential energy expressed as function of the same variables as for the differential equation. These integral minimisation methods were applied in the 19th century (for example, Rayleigh - Ritz method) for obtaining approximate solutions for vibrating systems. But with modern computers, these methods are applied to vibration of very complex and interconnected systems from molecules to aircraft and satellites. But there are limitations to applying these methods for dissipative systems which exhibit chaotic behaviour, for example, with turbulence and friction. Usually, there are no general integral constraints comparable to those that that exist for simpler systems, though hypotheses have been suggested that certain dissipative systems might minimise or maximise dissipation.

Optimisation methods have had their widest application in the management of organisations, especially in enabling them to meet a range of objectives with least resources and usually as fast as possible. Large organisations have networks of sub organisations distributed spatially. Planning the tasks of organisations and individuals, can, like computer programs, be represented as networks of sequential activities. The mathematical description and analysis of these space-time networks goes back to the $18^{\text {th }}$ century. By analysing his Sunday walks over all the bridges of Konigsberg in terms of a conceptual network of nodes, Euler showed in the 1740's whether it was or was not necessary to cross any bridge twice in order to cross all the bridges (Bondi, 1991). This began the generic mathematical description of shapes and curves on bodies with differing topological forms (for example, planes, spheres, doughnuts etc) in terms of edges, surfaces, singular points and how the shapes and curves do or do not change when the shapes and curves are distorted.(i.e. corners, junction of lines, centres of spirals etc.) (for example, Flegg, 1974). This analysis led engineers and scientists to developing very general and efficient methods for describing and classifying many types of real and conceptual patterns of curves and shapes. This application of simple topology can help explain the geometrical influences of the boundaries on processes such as flows through blood vessels, and around and through building complexes (Hunt et al., 1978).

In the 20th Century there was a growing interest in using network theory for planning resources and human activities while satisfying policy or economic constraints. The varied types of behaviour of such networks is modelled by sets of mathematical functions for example, calculating the total time to travel along a network depending on the path taken.

Mathematical methods were derived for calculating the optimum performance of both the linear and non-linear systems by minimising certain mathematical quantities derived from output variables for example, cost, speed etc. (for example,

Mathematical Modelling (ICTMA12) Education, Engineering and Economics ISBN 978-1-904275-20-6 Chichester: Horwood (2007) 510pp.

Editors: Christopher Haines, Peter Galbraith, Werner Blum and Sanowar Khan. 
Bellman \& Kalaba, 1965). The performance could be optimised 'on -line' as new data arrives. These developments came after World War II to coincide with the widespread use of electronic computers. They ensured the success of the hugely complex space exploration missions in the 1960's, with their on board 'minicomputers'.

In the emerging discipline of management sciences, these techniques contributed to the extraordinary improvements in the cost-effectiveness and greater reliability of construction projects and production systems ranging from engineering to agriculture.

With these optimisation and control methods, the frequency of failure in most operational systems has been steadily declining, such as in aviation and railways (for example, Evans, 2005).

By the 1980's in Europe and USA there was increasing interest in applying the concepts of system optimisation beyond their original well defined application in technology and production processes to the less well defined problem of optimising investment and strategy for individuals and corporations, and the management of very large commercial and governmental organisations.

Politicians and managers thought that targets for improved performance could be imposed on most types of organisation (for example, in terms of profit or the speed / quality/choice of certain deliverables). It was implicitly assumed, though seldom demonstrated, that the functioning of the organisations could be understood and even modelled well enough that the implications for reaching 'targets' could be known and had been worked out. Although the discipline of such targets doubtless improved certain aspects of the effectiveness of many organisations, analysis has shown that non-targeted outputs can suffer, for example in speed, quality and reliability.

Whether this was the reason or whether there was deliberate malfeasance (not in the model) some large international corporations and government agencies have actually or nearly failed operationally and/ or financially. The consequence is that governments now have to regulate organisations more closely, relying on external and transparent mathematical modelling to do so.

Clearly the models have to evolve to reflect the changing constraints and output variables, as well as the changing nature of organisations and their financial operations and (for example, Gallivan, 2006). Some of the most risky and profitable operations have been stimulated by the very fast processing of data made possible with modern computer systems.

However optimisation of systems is often limited in practice by the capacity and speed of available computers, since their complexity of system is growing so rapidly. Designing and/or predicting the performance of complex systems of continuous field variables (electromagnetic, fluid, solid, chemical etc) which are dependent on vast fields of data, are particularly demanding. In such modelling, even with the largest computational resources, the smallest scale processes, such as cracks in metals or turbulent eddies, have to be approximated in order to speed up the calculations or even to represent them at all. But approximations that smooth out local gradients can lead to large and sometimes chaotic errors; as occurred in the first experiments using numerical methods for weather forecasts. Understanding the qualitative nature of these errors has been a great break through in applied and

Mathematical Modelling (ICTMA12) Education, Engineering and Economics ISBN 978-1-904275-20-6 Chichester: Horwood (2007) 510pp.

Editors: Christopher Haines, Peter Galbraith, Werner Blum and Sanowar Khan. 
computational mathematics (for example, Broomhead \& Iserles, 1992).

Approximations are even more essential where calculations have to be repeated many times in order to optimise the accuracy for given input data or to find the optimum design by varying the design parameters of the system. As the capacity and speed of computers increase (beyond the present maximum level of $10^{14}$ operations per second of the Japanese 'Earth Simulator' systems) calculations are becoming progressively more elaborate. Input data and output predictions are more detailed and therefore more extensive in space and time, (typically $10^{9}$ units of input data per day are provided for numerical weather prediction systems) and more detailed processes are being modelled in ever more detail. Effectively there is a 'Parkinson's law' of computing capacity, i.e. the demands for more detail, larger domains and system complexity always outstrip the increase in capacity. This means that new approximations are always needed, as new processes and new constraints are introduced into the models. The use in global climate models of modern computing systems exemplifies these trends; more realistic simulations of aerosols and associated chemical processes are being incorporated and the duration of the model runs become ever greater as the demand grows to simulate prehistory and various, and sometimes alarming, scenarios for the distant future!

For optimisation of engineering designs, the aim of mathematical algorithms is primarily to provide efficient methods for calculating the effects on the performance of varying the design parameters, including possible variations in the shapes of the components. From nano-technology devices to buildings, even the topology of the system might be changed. The shapes of aeroplane wings have been optimised to minimum the aerodynamic drag given certain constraints on the shape of the wing (for example, lift force to carry the pay load, volume to carry fuel, strength etc.).

Since aircraft cruise at speeds close to the speed of sound, shock- waves form on the wings. However through optimised redesign of the wings on Boeing and Airbus aircraft, the position and amplitude of the shock waves were changed leading to substantial reductions (30-50\%) in the drag (for example, Jameson, 2000). The power of similar optimisation techniques should lead to quite new designs of integrated engineering systems and their more effective operation. Targets of 50\% to $70 \%$ reduction in automobiles' use of energy, and of an $80 \%$ decrease in ground level noise from aircraft are believed to be practical. Researchers and industrialists have argued that the pressure from government to make these environmental improvements needs to be stronger to make optimum use of current and emerging technological capabilities including those of mathematical modelling (Hunt, 2004).

Optimisation methods are particularly valuable for ensuring that computer models make best use of approximations and incomplete input data, even if it is not always reliable or accurate. These are critical issues for improving numerical predictions of weather and climate. .It was natural that this branch of computational mathematics should be the first to pioneer the methodology of calculating an 'ensemble' of solutions for a given model, using a range of input data corresponding to estimates of its incompleteness (for example, Hunt, 1999). The second pioneering innovation in complex computation probably resulted from the regular exchange of measurements around the world by meteorological services. They also exchange computational data, algorithms and 'advisories' to alert each other about extreme environmental conditions. Building on this tradition, these centres invented methods

Mathematical Modelling (ICTMA12) Education, Engineering and Economics ISBN 978-1-904275-20-6 Chichester: Horwood (2007) 510pp.

Editors: Christopher Haines, Peter Galbraith, Werner Blum and Sanowar Khan. 
of combining the results of different approximate models (using approximately the same input data). The results showed, as expected, a wider distribution of calculated solutions for the future weather. 'Outlying' predictions in the ensemble are usually made by one or two models. These have provided useful guidance about the possibility of extreme conditions (Harrison et al., 1999).

A less expected benefit of using wider ensembles is that the mean values of the ensemble predictions prove to be, in general, more accurate than those of any particular model (provided they are all of comparable level of resolution).

Calculations of an enormous ensemble of climate predictions are now also being run by thousands of interested members of the public using their own personal computers (climate.net, 2005). This is a new approach to engagement of the public in scientific policy issues; as well as providing valuable data on possible climate scenarios.

These consensus approaches to modelling are being applied to the socially important problems of predicting changes to global and local climates, especially the even more controversial problems of predicting and planning how to reduce the adverse effects of human activities.

It is not yet clear whether this emerging methodology of multi-model multicentre ensembles will be taken up in other fields where comparably large computer models and data inputs are needed for operational prediction, for example in geophysics, or engineering; or in modelling of biological systems. An alternative approach is being developed (Noble, 2002) for modelling the functioning of the healthy, and unhealthy, human heart; and how to deal surgically or pharmacologically with its defects. The various components (for example, nerve, fluid flow, walls of the vessels) are developed in different centres and then integrated into one 'super' model.

There is also enough open exchange of techniques and data in the field of geophysics, and enough urgency about the need for predictions that these collaborative methods could be tried to improve those models that have a credible level of predictability, such as those for tsunami waves, wide area flood forecasts (Hunt, 2005; Laguzzi et al., 2001), solar bursts and their interaction with the other magnetosphere (for example, Hunt \& Coates, 2003).

In engineering some of the largest computer modelling systems in the world have been constructed in the recent Accelerated Strategic Computing Initiative programme of the US government. One such system included all the components in an aero engine and another for a rocket (McMillan, 1999). Although these models use the best research from other centres they were not multi-centre models, but firmly located at particular universities (Stanford, Illinois). The lessons learnt were shared by other institutions of the department of Defence who had funded the project.

So far there are no plans in Europe to develop multi-centre computational models for engineering systems, even though this might be possible using the new GRID for very rapid data exchange. No 'grand challenge' projects have been emerged comparable to these in the USA (other than the continuation of the very successful human Genome project).

The development of large computer models for complex systems with wide social implications might be highly suitable projects. There could be further 
development of collaborative models of the human heart and other organs, because these show great promise for improving understanding and for guiding treatments. They might also reduce the requirement for human or animal experimentation that is necessary at present (House of Lords, 2003).

The future operation of transport and energy network systems, need to be improved because their reliability and safety is essential to a modern society (as events in Europe and USA since 2001 have painfully demonstrated). Currently, different elements of their performance are predicted by many organisations using different models and different inputs of data. Similar multi-centre ensembles of predictions could be combined using the approximate models operated by government agencies and commercial organisations, and data for the calculations that is incomplete (as much because of secrecy and slow communications as lack of measurements).

The same lack of collaboration afflicts economic planning and forecasting. As far as is possible to learn from public pronouncements, national and international government agencies and commercial organisations are each running their own predictive economic models.

It is surely likely that multi-model centres economic models will be tested before long, and may well become as useful as in weather/ climate prediction.

\section{CONCLUSION AND LOOKING FORWARD}

Lancelot Hogben's marvellous book 'Mathematics for the million' and many similar books across the world were aimed at popularising mathematics for the intellectually curious general public. Hogben (1936) particularly focused on the evolution of mathematical culture through the ages. Whereas this paper is addressed to those educating the millions of decisions takers across the world whose roles as senior managers, politicians, civil servants and university teachers involve them in applying the techniques of computing, modelling statistical analysis etc, but also using the broad ideas of mathematical science as. These ideas lie behind the ways that they reach their decisions and explain them. Consequently if journalists, politicians, and the public were more familiar with these concepts they would be able to question organisations more effectively. (In my experiences as a manager mathematical system concepts certainly gave some insight into how far ahead preplanning is likely to be possible before chaotic influences take over!)

Examples about the growing use of applied mathematics concepts have undoubtedly been helped by their popularisation and by progress in mathematical research (especially in dynamical systems). However, other powerful concepts which have not yet been so well popularised may well also have extensive use in practice. New pictorial images, which were so effective in stimulating interest in fractals and chaos, may be the most effective way to develop interest and understanding in the equally important themes of risk, sudden change, optimisation, new images are needed

Almost all of these concepts arose where mathematics was being applied in diverse fields of natural and social sciences and technology. The ever increasing speed and capacity of computers for calculations and for storing, sorting and transmitting of information of data systems are almost outpacing the rate at which 
research is producing new concepts for testing on the new computer and data systems. As we have shown, weather forecasts, the Genome project and modelling of biological system are introducing new approaches to modelling computation and interpretation. Perhaps government, research agencies and industry need to consider how these techniques and capabilities could be applied more widely for frontier research problem that are of great practical importance, such as natural disasters and complex socio-economic environmental systems.

Institutes and academic societies are exploring these new areas of mathematical modelling and also disseminating them through workshops, booklets and software, not only for instruction and sharing experience in modelling and computations (for example, Brown \& Leese, 2005). They have led to publications of guide-lines for the techniques and also for the strategy of modelling, for example, the appropriate application of modelling and measurement data in the controversial field of computational fluid dynamics and environmental prediction (for example, ERCOFTAC, 1998; Olsen 1998).

There seems to be a plenty of scope for decision makers and mathematical scientists to explore new ways in which the broad ideas of mathematics might be relevant, both in terms of particular techniques and in terms of strategic concepts for decision making about the many enormous problems facing the world.

Finally those teaching in universities might like to consider whether the 'big themes' of applied mathematics and statistics should be discussed in a few lectures to the final-year students in mathematical sciences before they leave, and to graduate students. With plenty of examples, this might encourage mathematical scientists to realise that their discipline fits them for the most responsible positions in societies, just as much as, say, the study of history or economics.

Also, providing such courses might stimulate more broad-minded mathematical scientists to share their insights with those based in other academic disciplines about how modern societies function and are organised. From my experience, few disciplines are as relevant to decision-making today as those of applied mathematics and statistics.

\section{ACKNOWLEDGEMENTS}

I am grateful to Chris Haines of City University for inviting me to lecture on this unusual topic, and to Frank Moon, Meghnad Desai, Steven Bishop, Steven Blinkhorn and other colleagues for their comments and insights, especially the visiting speakers at the LIMS evening lectures, and the political discussions of modelling in the House of Lords. This paper was partly written at Cornell University where I was Mary Upson visiting Professor in Mechanical Engineering.

\section{REFERENCES}

Allen, M. and Stainforth, D. (2002) Towards objective probabilistic climate forecasting, Nature 419, 228.

Armbruster, D. (2006) Private Communication. Arizona State University.

Ashford, O. (1985) Prophet or Professor; The life and work of L. F. Richardson. Bristol: Hilger. 
Belman, R. and Kalaba (1965) Dynamic programming and modern control theory. New York: Academic Press.

Bewley (2001) Flow control: new challenges for a new Renaissance. Aerospace Science, 37, 21-58.

Bondi, C. (ed) (1991) New applications of Mathematics. London: Penguin.

Bonnet, J.P., and Glauser, M.N., (1993) Eddy structure identification in free turbulent shear flows. Dordrecht: Kluwer Academic Publishing.

Broomhead D. S., and Iserles A. (eds) (1992) The dynamics of numerics and the numerics of dynamics. Oxford: Clarendon Press.

Brown, M. and Leese, R., (eds) (2005) New and emerging themes in industrial and applied mathematics. European Commission, Directorate-General for Research, Rep 21797.

Butler, A., Hefferman J.E., Tawn, J.A., Flather, R.A. and Horsburgh K. (2005) Recent trends in North Sea surge elevation. Elsevier Science pre-print.

Casulli V and Stelling G. (2001) Numerical simulation of the vertical structure of discontinuous flows. International Journal of Numerical Methods in Fluids, 37, 23-43.

Chalmers, A.F. (1982) What is this thing called science? Milton Keynes: Open University Press.

Colman A.W. and Davey, M.K. (1999) Prediction of summer temperature, rainfall and pressure in Europe from preceding winter North Atlantic Ocean temperature, International Journal of Climatology.

Cox, D.R., Isham, V.R. and Northrop, P.J. (2002) Floods: Some probabilistic and statistical approaches. Proceedings of the Royal Society A, 360, $1389-1408$.

Davies, T. and Hunt, J. C. R. (1995) New Developments in Numerical Weather Prediction. In K.W. Morton and M.J. Baines (eds) Proceedings of ICFD Conference on Numerical Methods for Fluid Dynamics V., Oxford: OUP, 1-18.

Embrechts, P., Klueppelberg, C. and Mikosch, T., (1997) Modelling Extremal Events: for Insurance and Finance. Applications of Mathematics. Berlin: Springer, vol. 33.

ERCOFTAC 2000 (2000) Best Practice Guidelines for industrial CFD. Available from Prof A. Hutton, Qinetiq, Farnborough UK.

Evans, A.W. (2003) Accidental fatalities in transport. Journal of the Royal Statistical Society, 166, 253-260.

Farge, M., Hunt, J.C.R. and Vassilicos, J.C., (eds) (1993) Wavelets, fractals and Fourier transforms. Oxford:Clarendon Press.

Favre, A. (ed ) (1995) Chaos and Determinism. Baltimore: Johns Hopkins Press.

Flegg, G. (1974) From Geometry to Topology. Milton Keynes: Open University Press

Gallivan, S. (2006) Is the National Health Service subject to hidden systems effects? LIMS Seminar, March 2006.

Garnett, J.C.M. (1921) Education and World citizenship. Cambridge: CUP.

Hacking I. (1975) The emergence of probability. Cambridge: CUP.

Haldane, J.B.S. (1935) Philosophy of a biologist. Oxford: Clarendon Press.

Harrison, M.S.J., Palmer, T.N., Richardson, D.S., Buizza, R. and Petroliagis, T. (1999) Analysis and model dependencies in medium-range ensembles: two transplant case studies. Quarterly Journal of Research Meteorological Society.

Mathematical Modelling (ICTMA12) Education, Engineering and Economics ISBN 978-1-904275-20-6 Chichester: Horwood (2007) 510pp.

Editors: Christopher Haines, Peter Galbraith, Werner Blum and Sanowar Khan. 
Heming, J.T., Chan, J.C.L. and Radford, A.M. (1995) A new scheme for the initialisation of tropical cyclones in the UK. Meteorological Office global model. Meteorological Applications 2, 171-184.

Hogben, L. (1936) Mathematics for the Million. London.

Holmes, P.J., Lumley, J.L. and Berkooz, G. (1996) Turbulence, Coherent Structures, Symmetry and Dynamical Systems. Cambridge: Cambridge University Press.

Houghton, J. L. (1994) Global Warming: The complete briefing. Oxford: Lion.

House of Lords (2002) Chips for everything. Select Committee Report. London: House of Lords.

House of Lords (2003) Scientific Experiment on Animals. Select Committee Report. London: House of Lords.

Howard, L. (1803) On the modifications of clouds. Essay to the Askesian Society. London.

Hunt, J.C.R., Abell, C.J., Peterka, J.A. and Woo, H.G.C. (1978 \& 1979) Kinematical studies of the flow around free or surface-mounted obstacles: applying topology to flow visualisation. Journal of Fluid Mechanics, 86, 179-200; corrigendum 95, 796.

Hunt, J.C.R. (1993) Life and Work of L.F. Richardson. In P. Drazin and I. Sutherland (eds) Collected works of L.F. Richardson. Cambidge: CUP, 1-27. (Also (1998) in Annual Review of Fluid Mechanics, 30.)

Hunt, J.C.R. and Neunzert, H. (1993) Mathematics and Industry. In: Proceedings of theEuropean Mathematics Congress Paris 1992. Birkhauser. (Also in (1994) IMA Bulletin, 29, 164-171.)

Hunt, J.C.R. (1995) Forecasts and warnings of natural disasters and the roles of national and international agencies. Meteorological Applications, 2, 53-63.

Hunt, J.C.R. (1997) Rounding and other approximations for measurement records and targets. Mathematics Today, 33, 73-77.

Hunt, J.C.R. (1999) Environmental forecasting and modelling turbulence. Physica D, 133, 270-295, (Proceedings of Los Alamos Conference on Predictability).

Hunt, J.C.R. (2001) Mathematical Model could clarify arms race. Nature, 411, 737.

Hunt, J.C.R. (2002) Floods in a changing climate. Philosphical Transactions of the. Royal Society, 360, 1531-1543.

Hunt, J.C.R. and Coates, A. (2002). Developments in space engineering and space science. Philosphical Transactions of the Royal Society, 361, 205-218. (See also Nature, 427, p13, 2004).

Hunt, J.C.R. and Coates, A. (2002) Developments in space engineering and space science. Philosophical Transactions of the Royal Society, 361, 205-218. (See also (2004) 'Joint efforts needed to forecast space weather' correspondence in Nature, 427, 13.

Hunt, J.C.R. (2004) Conclusions, Key Issues, Highlights $-13^{\text {th }}$ World Clean Air and Environmental Protection Congress and Exhibition. IUAPPA Newsletter, November, 2-4.

Hunt, J.C.R. (2005) Inland and coastal flooding: developments in prediction and prevention. Philosphical Transactions of the Royal Society A, 363, 1261-1491.

Hunt, J.C.R. (2005) Tsunami waves and coastal flooding. Mathematics Today.

Mathematical Modelling (ICTMA12) Education, Engineering and Economics ISBN 978-1-904275-20-6 Chichester: Horwood (2007) 510pp.

Editors: Christopher Haines, Peter Galbraith, Werner Blum and Sanowar Khan. 
Huppert, H.E. and Sparks, S. (2006) Proceedings of the Royal Society Conference on Natural Disasters. Philosphical Transactions of the Royal Society A, August.

International Strategy for Disaster Reduction (2005) Kobe International Conference. Geneva: United Nations.

Jameson A. and Alonso J.J. (2000) Future Research Avenues in Computational Engineering and Design. Proceedings of ICIAM 99. Oxford: OUP.

Kelly, F.P. (1979) Reversibility and Stochastic Networks. Chichester: Wiley.

Krishnamurthi, R. and Howard, L. (1981) Large-scale flow generation in turbulent convection. Proceedings of the National Academy of Sciences USA, 78, 19811985.

Landau, L. and Lifśhitz, L. (1960) Statistical Physics. Pergamon.

Lautso K, Spiekermann, K. Wegener M, Sheppard I, Steadman P, Martino A, Domingo R and Gayda S. (2004) PROPOLIS: Planning and Research of Policies Land Use and Transport for Increasing Urban Sustainability. Brussels: European Commission.

Lighthill, M.J. (1953) Generalised functions Cambridge: CUP.

Lighthill, M.J. and Whitham, G.B. (1955) On kinematic waves. II. Theory of Traffic. Flows on Long Crowded Roads. Proceedings of the Royal Society A, 229, 317-345.

Lorenz, E.N. (1963) Deterministic non-periodic flow. Journal of Atmosphere Science, 20, 130-141.

Mandelbrot, B. (1982) The fractal geometry of nature. San Francisco: Freeman.

McMillan, C. (1999) Introduction to the Accelerated Strategic Computing Initiative Academic Strategic Alliance Program (Lawrence Livermore National Laboratory, Livermore, Ca) AIAA Fluid Dynamics Conference, $30^{\text {th }}$, Norfolk, VA, June 28-July 1.

Moon, F. C. (2004) Chaotic Vibrations. New Jersey: Wiley.

Nikiforakis, N., and Hubbard, M.E. (2003) A three-dimensional, adaptive, Godunov-Type Model for Global Atmospheric Flows. Monthly Weather Reviews, 131.

Noble, D. (2002) Modelling the heart: from genes to cells to the whole organ. Science, 295, 1678-1682. Merlin Press.

Oslen, H.R. (1998) Local scale regulatory dispersion models; initiatives to improve modelling culture. $10^{\text {th }}$ Conference on Applications and Air Pollution in Meteorology. Boston: American Meteorological Society.

Poincaré H. (1914) Science \& Method (English translation) (Preface by Bertrand Russell) London: Nelson.

Reuleaux F (1885) The influence of the Technical Sciences upon General Culture. School of Mines Quarterly VII(1), October.

Richardson, L.F. (1922) Weather prediction by numerical process. Cambridge: CUP.

Saunders, M.A. and Lea, A.S. (2005) Seasonal prediction of hurricane activity reaching the coast of the United States. Nature, 434, 1005-1008.

Saunders, P.T. (1980) An introduction to Catastrophe Theory, Cambridge: CUP.

Schellnhuber, H.J., Crutzen, P.J., Clark, W.C. and Hunt, J.C.R. (2005) Earth system analysis for sustainability. Environment, 47(8), 10-27. 
Shannon, C.E. and Weaver (1949) The mathematical theory of communication Urbana: University of Illinois Press.

Silverman, B.W. and Vassilicos, J.C. (ed.) (1999) Wavelets: the key to intermittent informations. London: Royal Society.

Spearman, C. (1904) General Intelligence, Objectively Determined and Measured. American Journal of Psychology, 15, 205-260.

Thompson, J.M.T. and Stewart, H.B. (2002) Non-linear Dynamics and Chaos. London: Wiley.

Thomson, W. and Tait, P.G. (1879) Treatise on Natural Philosophy, Part 1. Cambridge: CUP.

Turfus, C. (2006) Mathematical tools in finance. Mathematics Today, April.

Volterra, V. (1931) Lecons sur la théorie mathématique de la lutte pour la Vie. Paris: Gauthier-Villars.

Zeeman E.C. (1987) On the Psychology of a Hijacker: In P.G. Bennett (ed) Analysing Conflict and its Resolutions. Oxford OUP. 